\title{
ASTEROID DETECTION FROM PIONEERS F AND G?
}

\author{
ROBERT K. SOBERMAN \\ General Electric Space Sciences Laboratory and Drexel University \\ and \\ SHERMAN L. NESTE AND ALAN F. PETTY \\ General Electric Space Sciences Laboratory
}

\section{INSTRUMENT DESCRIPTION}

The Pioneer asteroid/meteoroid detector (Sisyphus ${ }^{1}$ or $\mathrm{A} / \mathrm{MD}$ ) is an optical instrument designed primarily to make measurements of small interplanetary particles that pass within about $1 \mathrm{~km}$ of the spacecraft. Because it is an optical system, it can also detect larger bodies at greater distances. We can approximate the amount of light incident upon the instrument resulting from an assumed spherical object by

$$
I=\frac{I_{0} r \pi a^{2}}{2 s^{2} \pi R^{2}}=\frac{I_{0} r}{2 s^{2}}\left(\frac{a}{R}\right)^{2}
$$

where $I_{0}$ is the solar irradiance at $1 \mathrm{AU}, r$ is the reflectivity of the object (equal to $3 / 2$ the "geometric albedo"), $a$ is the radius of the object, $s$ is the distance from the Sun in astronomical units, and $R$ is the range from the object to the detector. A Sun-object-instrument angle of approximately $45^{\circ}$ is assumed. For a single detector, one would have no way of distinguishing objects with the same $a / R$ ratio. The Sisyphus concept provides a means of determining the range and, hence, the size of the object.

Consider three optically alined telescopes equipped with photomultipliers as defining three parallel cones in space. If the telescopes are identical, then the edges of the fields of view remain at a fixed distance from each other regardless of range. Any luminous object that crosses through the intersecting fields of view is then detected by each of the photomultipliers. From the entrance and exit times in each field of view, one can completely calculate the trajectory of the object in space, provided only that one has sufficiently good optics and a sufficiently long baseline between telescopes.

\footnotetext{
${ }^{1}$ Refers to man's never-ending confrontation with the environment; i.e., space rocks.
} 
For the mathematics of the system, we define three cones as shown in figure 1 ; their half angles are $\theta$. Lines joining their apexes form an arbitrary triangle in the plane perpendicular to their axes. For purposes of convention, the vector from the base of the $i$ th cone to the particle's entrance into that cone is designated $\boldsymbol{\rho}_{i}$ and the vector to the particle's exit is $\boldsymbol{\sigma}_{i}$. Times of entrance and exit at the $i$ th cone are designated $\tau_{\mathrm{ij}}$ where $j$ is 1 for an entrance point or 2 for an exit point. $\mathbf{v}$ is an arbitrary velocity vector and $l_{i j}$ is the distance between the $i$ th and $j$ th cone.

Using this convention, five independent vector equations result:

$$
\left.\begin{array}{l}
\sigma_{1}=\rho_{1}+\left(\tau_{12}-\tau_{11}\right) v \\
\rho_{2}=\rho_{1}+\left(\tau_{21}-\tau_{11}\right) v-l_{12} \\
\sigma_{2}=\rho_{1}+\left(\tau_{22}-\tau_{11}\right) v-l_{12} \\
\rho_{3}=\rho_{1}+\left(\tau_{31}-\tau_{11}\right) v-l_{13} \\
\sigma_{3}=\rho_{1}+\left(\tau_{32}-\tau_{11}\right) v-l_{13}
\end{array}\right\}
$$

By breaking these into components, we have 15 equations in 15 unknowns; therefore, a solution exists. Because the derivation is long and tedious, it is omitted here. The solution has been programed for computer use.

The above vector equations remain unchanged if the cone axes are misalined (i.e., not parallel). However, the 15 component equations are more complex

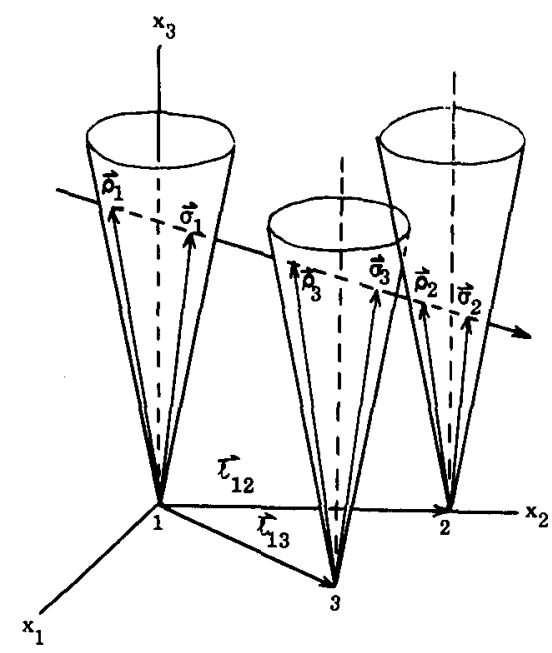

Figure 1.- Sisyphus geometry (for convention only). 
because they involve two additional angles for each cone necessary to specify its orientation. This misalined case has been reduced from the 15 original equations to 3 equations in 3 unknowns. Because of their complexity, further reduction appears impractical. Numerical solutions are obtained by computer iteration.

Thus, independent of the amplitude of the signals detected by the individual optical systems, one can establish the three velocity components and the range of the body. Using this calculated range, the measured light intensity at the detector, and the known solar intensity, one can solve equation (1) for the product of the reflectivity and the cross-sectional area, and thus determine the mean radius of the body to an uncertainty of the square root of the reflectivity. Further, from the real time at which the event took place; the known position, velocity, and orientation of the vehicle from which the measurement was made; and the three velocity components of the body, the complete orbit of the body in the solar system can be determined.

The $A / M D$, as designed for the Pioneer F and $G$ missions, uses four $20 \mathrm{~cm}$ Cassegrainian telescopes, each coupled to a photomultiplier tube (S20 photocathode) as sensor. The four optics are arranged in a square array $22 \mathrm{~cm}$ on a side. The use of four telescopes yields an inherent redundancy because any three sensors yield all of the required data. If a particle exceeds the threshold in all four fields of view, one obtains four sets of solutions to equation set (2). An artist's sketch of the A/MD instrument on the Pioneer spacecraft is shown in figure 2 . The instrument hardware (excluding spacecraft mounting panel) has a mass of $2.4 \mathrm{~kg}$ and an average power requirement of $2.0 \mathrm{~W}$.

The $\mathrm{A} / \mathrm{MD}$ is a background-limited detector. The noise inherent in such a detector is given by

$$
i_{n}=\sqrt{2 i_{b} q f}
$$

where $i_{b}$ is the total background current, $q$ is the unit electrical charge, and $f$ is the bandwidth of the circuitry

Because the Pioneer is a rotating spacecraft, the sky background viewed by the telescopes is continuously varying. The threshold in each telescope is designed to "follow" the background and high-frequency noise. Because the telescopes remain approximately alined, the background and, consequently, the thresholds of all four telescopes should remain approximately equal. The background is averaged through a comparatively long time constant circuit that has the effect of introducing a delay in the background response. The relative threshold for each telescope at any instant $t$ is designed to be self-setting at a value of

$$
T_{\text {rel }}=K_{1} i_{n}(t)+K_{2} i_{b}(t)-i_{b}(t-\Delta t)
$$

The operating modes of the instrument with the values for $K_{1}, K_{2}$, and the time constant $\Delta t$ for background averaging are given in table I. A computer 


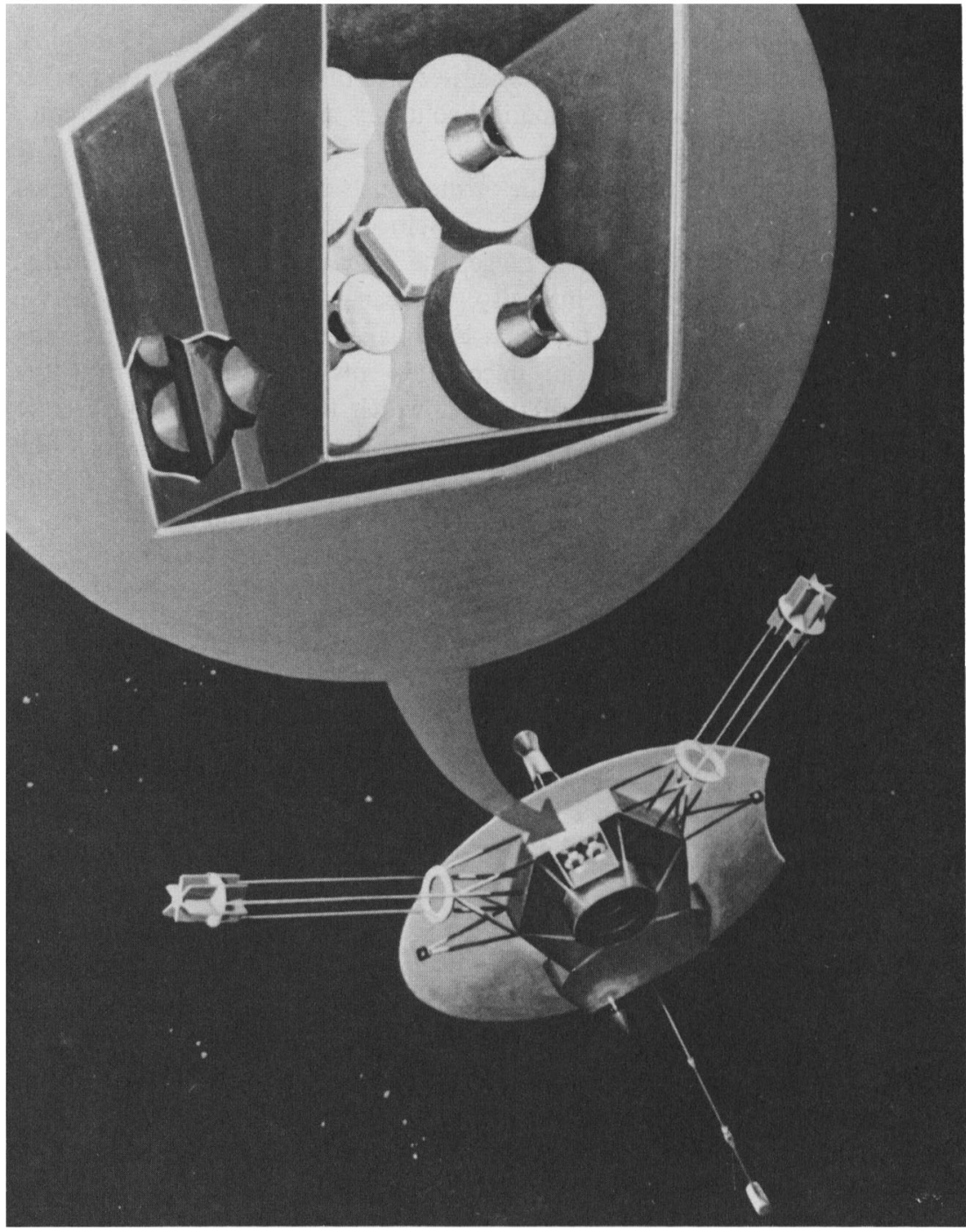

Figure 2.-Artist's sketch of Pioneer F and G A/MD.

program that calculates the change in the relative threshold over a complete spacecraft rotation as a function of the orientation of the spacecraft spin axis has been written (Soberman and Neste, 1971). The program has a sky brightness map (Roach and Megill, 1961) built in. A typical output for the wideband instrument mode is shown in figure 3.

Because the instrument is "triggered" by noise when the intensity exceeds the threshold value, a technique for reducing the number of false events must 
TABLE I.-Modes of Pioneer F and G Sisyphus Instrument

\begin{tabular}{|c|c|c|c|c|}
\hline $\begin{array}{l}\text { Operating } \\
\text { mode }\end{array}$ & $\underset{\mathrm{kHz}}{\text { Bandwidth } f \text {, }}$ & $K_{1}$ & $K_{2}$ & $\begin{array}{c}\text { Time } \\
\text { constant, s }\end{array}$ \\
\hline Wideband & 500 & 2.0 & 1.1 & 0.047 \\
\hline Mediumband & 160 & 2.0 & 1.1 & .047 \\
\hline Narrowband & 13 & 2.0 & 1.1 & .047 \\
\hline High threshold & $\left({ }^{a}\right)$ & 2.0 & 1.2 & .047 \\
\hline Calibration & 13 & 2.0 & 1.1 to 1.2 & .5 \\
\hline
\end{tabular}

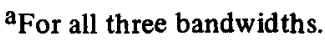

be employed. This is accomplished by a simple threefold coincidence requirement that must be fulfilled before an event is considered legitimate and is recorded. This means that the transit time of a particle through the overlap region of three of the four fields of view must be greater than or equal to a predetermined minimum $(3.2 \mu \mathrm{s})$. This criterion limits the number of recorded false events to approximately one per month for the wideband mode.

In addition, the thresholds are designed to have hysteresis to reduce the probability of rejecting a legitimate signal. For example, if a target is passing through the field of view and noise or fluctuations in target intensity cause the level to drop below the threshold before coincidence is confirmed, the signal would be regarded as an error and rejected. However, if the threshold is reduced to a lower level after being exceeded, the probability of dropouts due

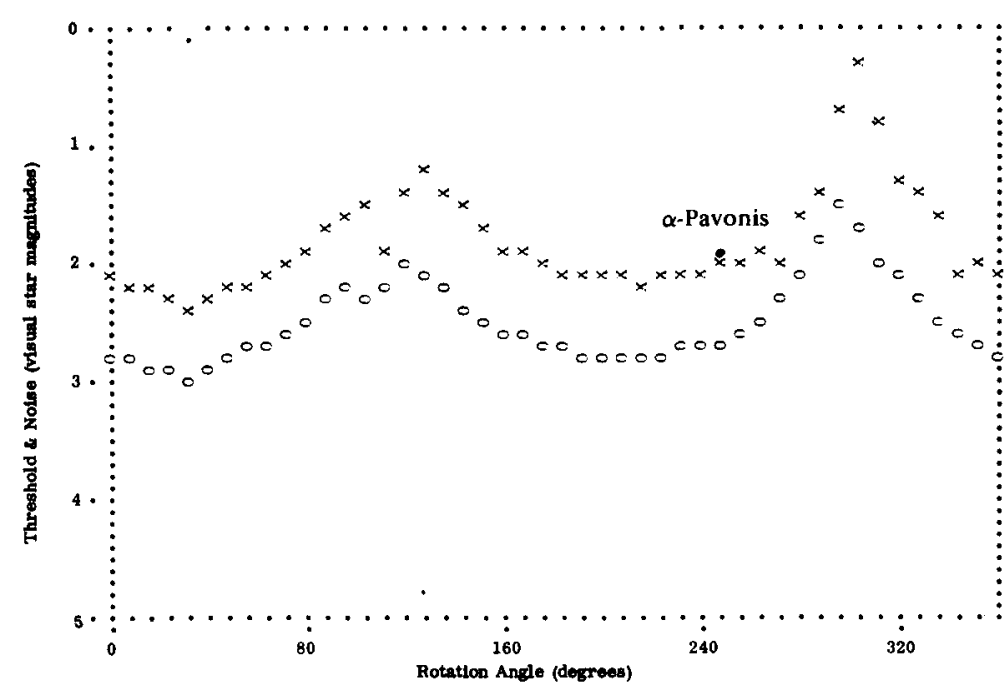

Figure 3.-Computer-generated cyclic threshold variation for Pioneer F and G A/MD in the wideband mode. Galactic longitude $=333^{\circ}$; galactic latitude $=0^{\circ}$; bandwidth $=500 \mathrm{kHz} . x$ indicates threshold; $\circ$ indicates noise. 
to noise is reduced. The rate at which the threshold is reduced and the level to which it is reduced do not affect noise rejection nor prevent a legitimate end of signal determination.

Because the threshold follows the background, a distant large asteroid moving with low relative angular velocity will, in several time constants of the averaging circuit, be considered as part of the background. When this happens, a false termination of the signal will occur. Another problem relating to the measurement of relatively slow-moving asteroids is the star exclusion circuit of the A/MD. To limit the amount of data telemetered, any "event" that recurs on successive vehicle rotations is ignored after the first measurement. Thus, if such a relatively slow-moving asteroid is detected, it can only be measured once in a given region of the sky. Operation in the "star exclusion disabled" mode, which would allow multiple measurements of the passage of slow-moving asteroids through the complete field of view, is incompatible with the present Pioneer A/MD telemetry assignment. Modifications of that assignment were considered too costly in view of the low probability of such measurements. (See below.)

\section{ASTEROID BELT MODELS}

Current models of the particle number density within the asteroid belt are usually expressed by a relationship of the form

$$
N(a)=C a^{-\alpha}
$$

where $a$ is the particle radius and $C$ and $\alpha$ are constants. The major uncertainty, and point of controversy, between the various models lies in determining the value of $\alpha$. Arguments for the various values of $\alpha$ are usually based on theoretical studies regarding the collision and subsequent fragmentation of particles within the beits. On the basis of such a grinding mechanism, Piotrowski (1953) argues that particles near $a=1 \mathrm{~cm}$ should follow an $\alpha=3$ law. However, Anders (1965) does not believe that the fragmentation history of the asteroids has progressed as far as does Piotrowski and favors a value of $\alpha$ more nearly equal to 2 .

A more recent model of the asteroid distribution is that published by Dohnanyi (1969) in which he considers the evolution of a system of particles undergoing inelastic collisions and fragmentation. ${ }^{2}$ He derives a theoretical density function for asteroids given by

$$
f(m) d m=2.48 \times 10^{-19} m^{-1.837} d m
$$

where $m$ is the particle mass. After performing the integration and expressing the number density in the form of equation (5), we obtain

$$
N(a) \approx 9.7 \times 10^{-23} a^{-2.5}
$$

${ }^{2}$ See p. 263. 
where $a$ is the particle radius in meters. Following Dohnanyi, a particle density of $3.5 \times 10^{3} \mathrm{~kg} / \mathrm{m}^{3}$ was assumed in the conversion from mass to radius.

Figure 4 presents some recent data on the cumulative asteroid distribution. The histograms are for the McDonald survey (Kuiper et al., 1958) and the more recent Palomar-Leiden survey (PLS) (van Houten et al., 1970). Distributions are shown for power-law exponents of -3 (Piotrowski), -2.5 (Dohnanyi), -2 (Anders), and the literal interpretation of the PLS of -1.75 . Implicit in

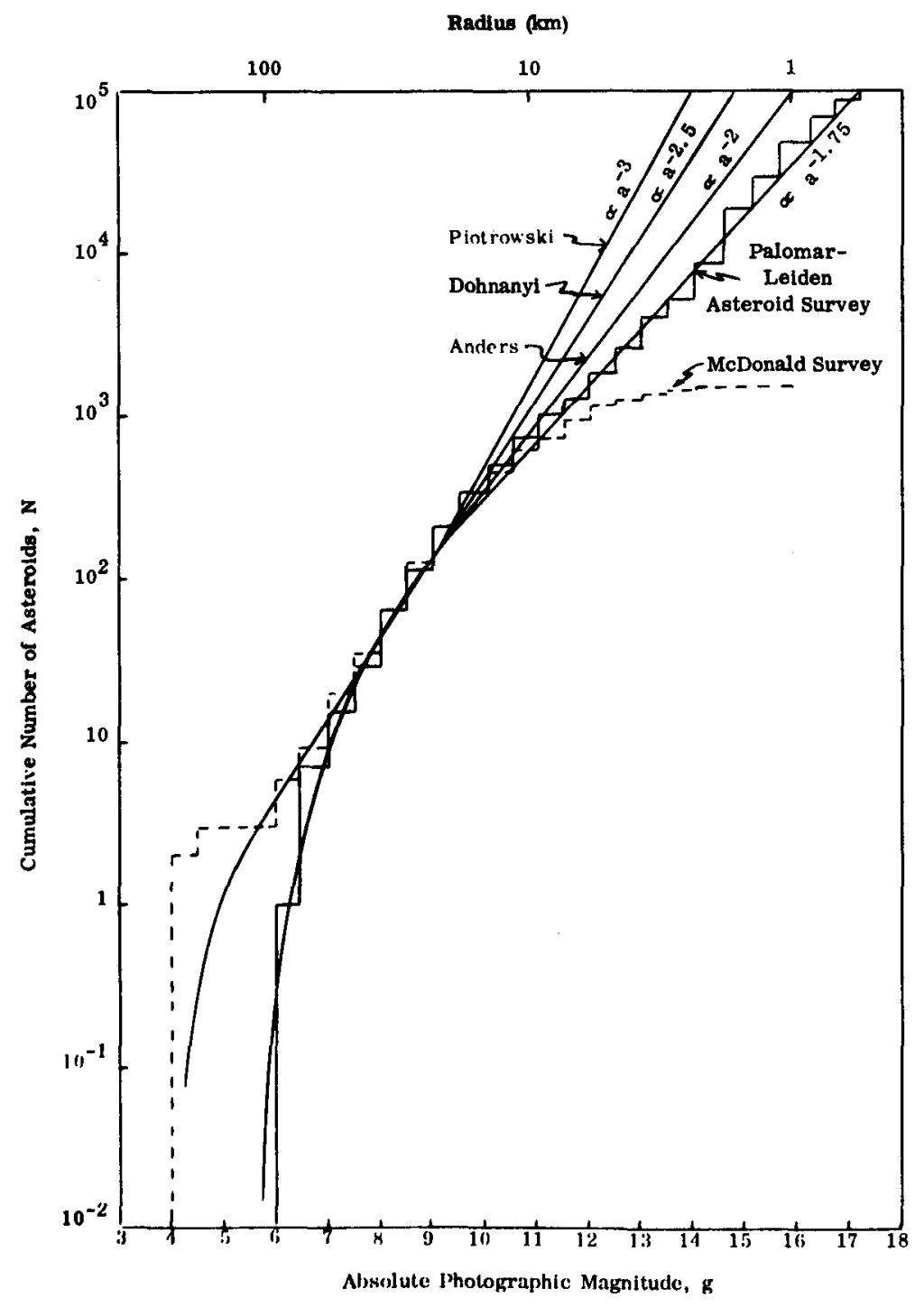

Figure 4.-Cumulative asteroid distribution. 
accepting any of the three steeper values is the assumption of a systematic selection process in the surveys. How far any of these extrapolations can be extended is problematical. For a portion of the present analysis it is necessary to extrapolate beyond the point of any reasonable confidence. However, until the Pioneer spacecraft have actually penetrated the asteroid belts, these extrapolations form the only source from which anticipated data rates can be inferred.

If we assume an average asteroid velocity of $15 \mathrm{~km} / \mathrm{s}$ relative to the Pioneer spacecraft, a cumulative asteroidal flux can be calculated for each of the

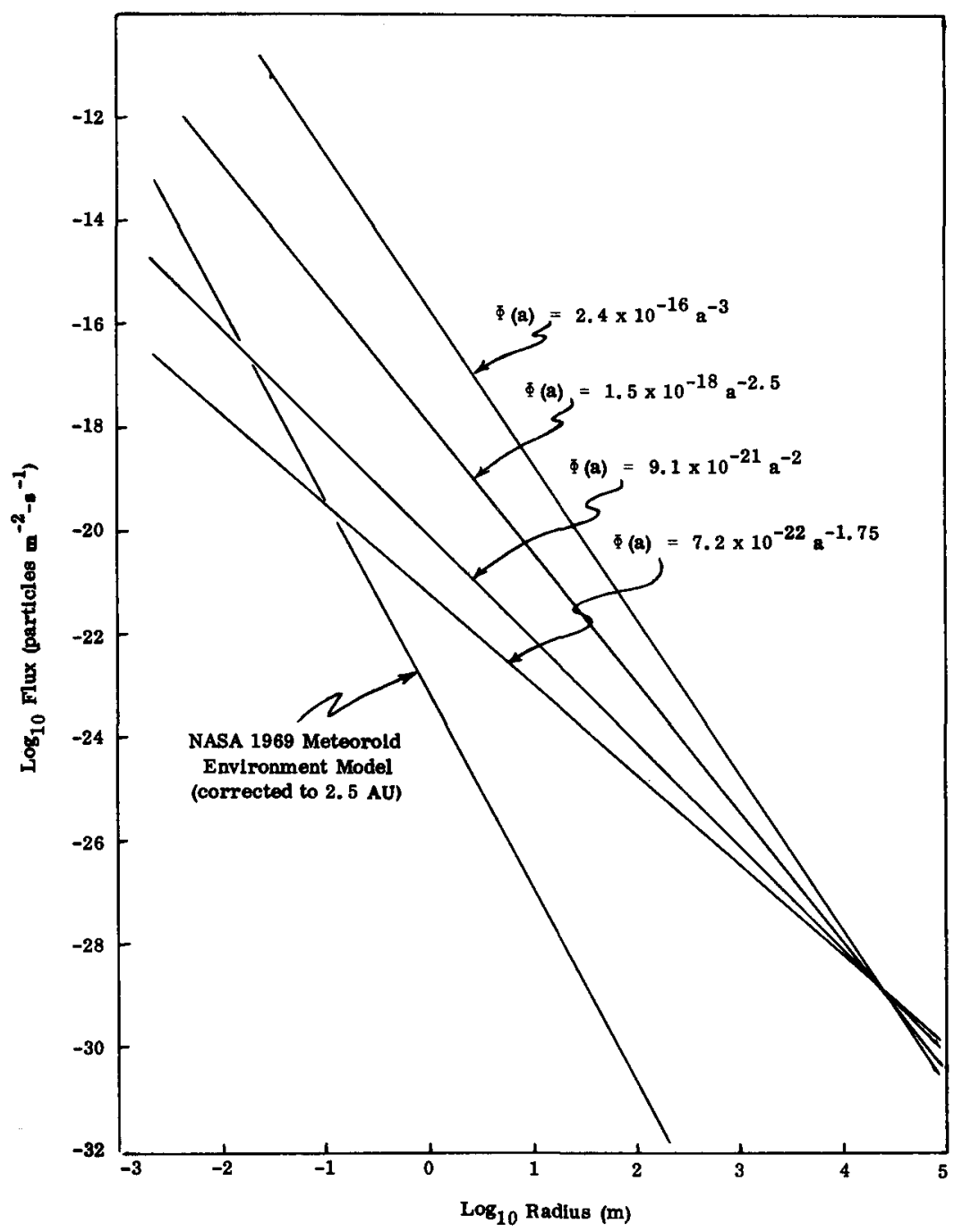

Figure 5.- Asteroid and cometary meteoroid flux models at $2.5 \mathrm{AU}$. 
distribution models. These are presented in figure 5 for the four models, together with a flux model for the meteoroid environment at $2.5 \mathrm{AU}$. The meteoroid environment was taken from NASA SP-8013 (1969), corrected for the Earth's focusing effect and reduced by an inverse-square dependence on the heliocentric distance.

Estimates of particle reflectivity are also open to speculation. For the present purposes, three values have been chosen. As pointed out above, the reflectivity used is equal to $3 / 2$ the geometric albedo. The three values used are a reasonable lower limit of 0.07 , a meteoroid value of 0.20 , and the mean for the asteroids Ceres, Pallas, Juno, and Vesta (Gehrels, 1970), which is 0.3. This last was converted from a geometric albedo of 0.2 (the value used by Dohnanyi, 1969).

\section{ASTEROID EVENT RATES}

The anticipated $\mathrm{A} / \mathrm{MD}$ event rates during the Pioneer transit of the asteroid belts can be determined for the various power-law models and particle reflectivities as shown below.

The area surrounding the field of view of the A/MD can be approximated as a truncated cone:

$$
A \approx \pi \theta\left(R^{2}-R_{\min }^{2}\right)
$$

and

$$
d A \approx 2 \pi \theta R d R
$$

for small $\theta$ (the half angle of the telescopes). In the wideband mode, it was shown in figure 3 that the mean sensitivity of the A/MD instrument is approximately 1.75 visual stellar magnitudes. This can be translated through equation (1) in terms of a range to radius relationship applicable at a heliocentric distance of $2.5 \mathrm{AU}$ :

$$
R / a=1.5 \times 10^{5} r^{1 / 2} \quad s=2.5
$$

Substituting this into equation (8), the effective detector area as a function of particle radius can then be expressed as

$$
d A(a)=1.4 \times 10^{11} \text { ora da }
$$

The asteroid number density can be written as

$$
N(a)=8.7 \times 10^{-34}\left(\frac{a}{a_{0}}\right)^{-\alpha}
$$


and converted to a particle flux per unit area with the assumption of an average particle velocity $v$ :

$$
\Phi(a)=8.7 \times 10^{-34}\left(\frac{a}{a_{0}}\right)^{-\alpha} v
$$

The event rate $E$ is then determined from the integral

$$
\begin{aligned}
E & =\int_{A} \int_{\Phi} d \Phi d A=\int_{A} \Phi d A \\
& =1.2 \times 10^{-22} \theta r v a_{0}{ }^{\alpha} \int_{a_{\min }}^{a_{0}} a^{1-\alpha} d a
\end{aligned}
$$

For $\alpha \neq 2$,

$$
E=1.2 \times 10^{-22} \frac{\theta r \nu}{2-\alpha}\left(a_{0}^{2}-a_{0}{ }^{\alpha} a_{\min }^{2-\alpha}\right)
$$

and for $\alpha=2$,

$$
E=1.2 \times 10^{-22} \theta r v a_{0}^{2} \ln \frac{a_{0}}{a_{\min }}
$$

Using the values

$$
\begin{aligned}
\theta & =4.0^{\circ} \\
v & =1.5 \times 10^{4} \mathrm{~m}-\mathrm{s}^{-1} \\
a_{0} & =2.6 \times 10^{4} \mathrm{~m} \\
a_{\text {min }} & =10^{-4} \mathrm{~m}
\end{aligned}
$$

for $\alpha \neq 2$,

$$
E=1.3 \times 10^{-19}\left[6.8 \times 10^{8}-10^{4 \alpha-8}\left(2.6 \times 10^{4}\right)^{\alpha}\right] \frac{r}{2-\alpha}
$$

and for $\alpha=2$,

$$
E=1.7 \times 10^{-9} r
$$

From this, we can obtain an event rate for each set of assumptions.

Because the $\mathrm{A} / \mathrm{MD}$ is oriented at an angle of $45^{\circ}$ from the spacecraft (S/C) spin axis, its field of view will describe a $49^{\circ}$ right cone in space of wall width approximately $8^{\circ}$. (See fig. 6.) Any particle that takes more than approximately $12 \mathrm{~s}$ (the vehicle rotation period) to pass through the $8^{\circ}$ instrument field of view must be seen by the system (i.e., it cannot escape from the 


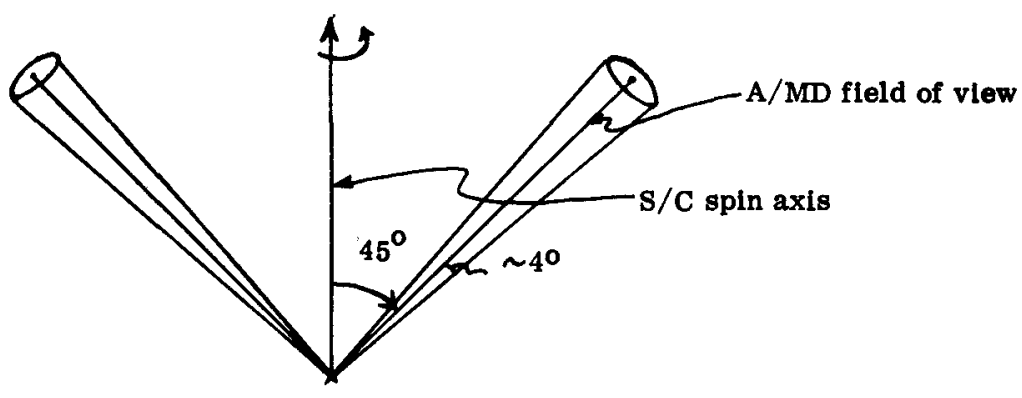

Figure 6. - Effective viewing field for large asteroids (angular velocity $<0.68 \mathrm{deg} / \mathrm{s}$ ).

$45^{\circ}+4^{\circ}$ cone described during the vehicle rotation without being detected). Thus, for large bodies moving at low relative angular velocity, the effective field of view becomes $98^{\circ}$. As an example, for a minimum asteroid size (radius) of $10 \mathrm{~m}$ and a reflectivity of 0.2 , the effective detection range is $650 \mathrm{~km}$ in the wide bandwidth mode. Event rates can thus be calculated for large asteroids using the effective cone area measured in square meters.

$$
\begin{aligned}
A & =\pi R^{2} \sin 49^{\circ} \\
& =5.3 \times 10^{10} a^{2} r \quad s=2.5
\end{aligned}
$$

This yields event rates of

$$
\begin{aligned}
E & =9.2 \times 10^{-23} \frac{r \nu}{2-\alpha}\left(a_{0}^{2}-a_{0} \alpha^{2} a_{\min }^{2-\alpha}\right) \quad \alpha \neq 2 \\
& =9.2 \times 10^{-23} r v a_{0}^{2} \ln \frac{a_{0}}{a_{\min }} \quad \alpha=2
\end{aligned}
$$

where $a_{\mathrm{min}}$ is now equal to $10 \mathrm{~m}$. The computations have been carried out for the combinations of $\alpha$ and $r$ to yield the event rates shown in tables II and III.

From tables II and III and from the nature of the $\mathrm{A} / \mathrm{MD}$, it is apparent that for power-law distributions steeper than those with $\alpha=2$, the event rate would be dominated by small particles. Although larger bodies would tend to predominate for power-law distributions with $\alpha=2$ or less, it is unlikely that the A/MD would detect any asteroid events. In point of fact, if $\alpha$ is much less than 2.5 , it is possible that passage of the Pioneer spacecraft through the asteroid belt would not even be noted. In figure 7 , we have plotted the anticipated cometary meteoroid event rate as a function of heliocentric distance. The values from table II are shown in the asteroid region. Included also is a maximum value based upon a zodiacal light interpretation (Kessler, 
TABLE II.-Asteroidal Events Per Day, Wideband Mode

\begin{tabular}{c|c|c|c|r}
\hline \multirow{2}{*}{$r$} & \multicolumn{4}{|c}{$\alpha$} \\
\cline { 2 - 5 } & 1.75 & 2.0 & 2.5 & 3.0 \\
\hline $0.07 \ldots \ldots \ldots$ & $2.1 \times 10^{-6}$ & $1.0 \times 10^{-5}$ & $1.7 \times 10^{-2}$ & 140 \\
$0.2 \ldots \ldots \ldots$ & $6.0 \times 10^{-6}$ & $2.9 \times 10^{-5}$ & $4.8 \times 10^{-2}$ & 400 \\
$0.3 \ldots \ldots \ldots$ & $9.0 \times 10^{-6}$ & $4.4 \times 10^{-5}$ & $7.3 \times 10^{-2}$ & 600 \\
\hline
\end{tabular}

Angular velocity $>0.685 \mathrm{deg} / \mathrm{s}$.

TABLE III.-Large Asteroid (a > 10 m) Events Per Day, Wideband Mode

\begin{tabular}{c|c|c|c|c}
\hline \multirow{2}{*}{$r$} & \multicolumn{4}{|c}{$\alpha$} \\
\cline { 2 - 5 } & 1.75 & 2.0 & 2.5 & 3.0 \\
\hline $0.07 \ldots$ & $2.3 \times 10^{-6}$ & $4.5 \times 10^{-5}$ & $7.1 \times 10^{-4}$ & $1.5 \times 10^{-2}$ \\
$0.2 \ldots$ & $6.5 \times 10^{-6}$ & $1.3 \times 10^{-4}$ & $2.0 \times 10^{-3}$ & $4.2 \times 10^{-2}$ \\
$0.3 \ldots$ & $9.7 \times 10^{-6}$ & $1.9 \times 10^{-4}$ & $3.0 \times 10^{-3}$ & $6.3 \times 10^{-2}$ \\
\hline
\end{tabular}

Angular velocity $<0.685 \mathrm{deg} / \mathrm{s}$.

1968). From this figure, one can see how the cometary flux might predominate during asteroidal passage. For small particles, the velocity determinations of the A/MD should, however, enable us to distinguish cometary from asteroidal material if both are noted.

Assuming a uniform distribution in the asteroid belt, the probability of the A/MD detecting a single large asteroid $(a \geqslant 10 \mathrm{~m}$ ) during the entire passage can be calculated from table III. The results are presented in table IV. Although the extrapolation is more reasonable in this limited size region, it is obvious from table IV that the distribution slope still dictates the probability of detecting a large asteroid.

The apparent magnitudes of 1735 asteroids that the Pioneer F spacecraft might encounter have been computed (NASA PT-204, 1970). For the vehicle trajectory ephemerides, which were used in that study, the brightest apparent magnitude anticipated is approximately 6.5. As shown above, the mean threshold for the wideband of the $\mathrm{A} / \mathrm{MD}$ is about 1.75 . Consequently, it is unlikely that any of the asteroids considered would be detected. However, as stated in the study, the results are "subject to a very large uncertainty."

One way of increasing the probability of large-body detection would be to narrow the bandwidth of the $\mathrm{A} / \mathrm{MD}$. In the narrowband mode, the mean threshold would be approximately 2.75 visual stellar magnitudes. (See fig. 8.) In this mode, linear velocity determination for small particles would be extremely poor at best. However, for large-body detection beyond the effective triangulation range of the Pioneer A/MD version, those consequences are 


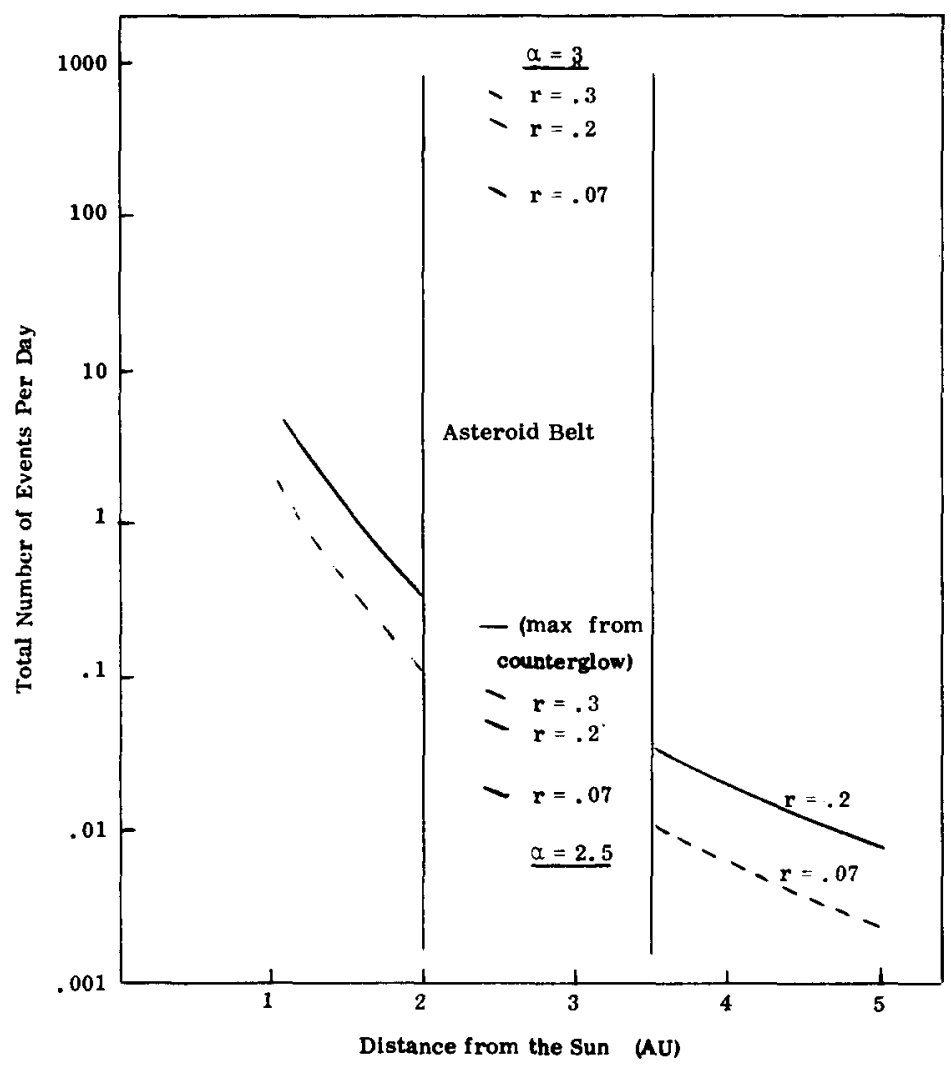

Figure 7.-Anticipated cometary meteoroid event rate for Pioneer $F$ and $G$ A/MD as a function of heliocentric distance with inferred asteroidal event rates.

TABLE IV.-Probability of Detecting a Large Asteroid (a $>10 \mathrm{~m}$ ) During a Mission, Wideband Mode

\begin{tabular}{r|r|r|r|r}
\hline \multirow{2}{*}{$r$} & \multicolumn{5}{|c}{$\alpha$} \\
\cline { 2 - 5 } & \multicolumn{1}{|c|}{1.75} & 2.0 & \multicolumn{1}{c}{2.5} & \multicolumn{1}{c}{3.0} \\
\hline $0.07 \ldots$ & 0.03 & 0.7 & 10 & 89 \\
$0.2 \ldots$ & .10 & 1.9 & 26 & $\sim 100$ \\
$0.3 \ldots$ & .15 & 2.8 & 33 & $\sim 100$ \\
\hline
\end{tabular}

Values are in percent.

immaterial. Should there prove to be a lack of particles within the wideband threshold capability, the option of switching to narrowband would be exercised. This option may also be exercised if representative data have already been obtained for the small particles. The result of switching to the 


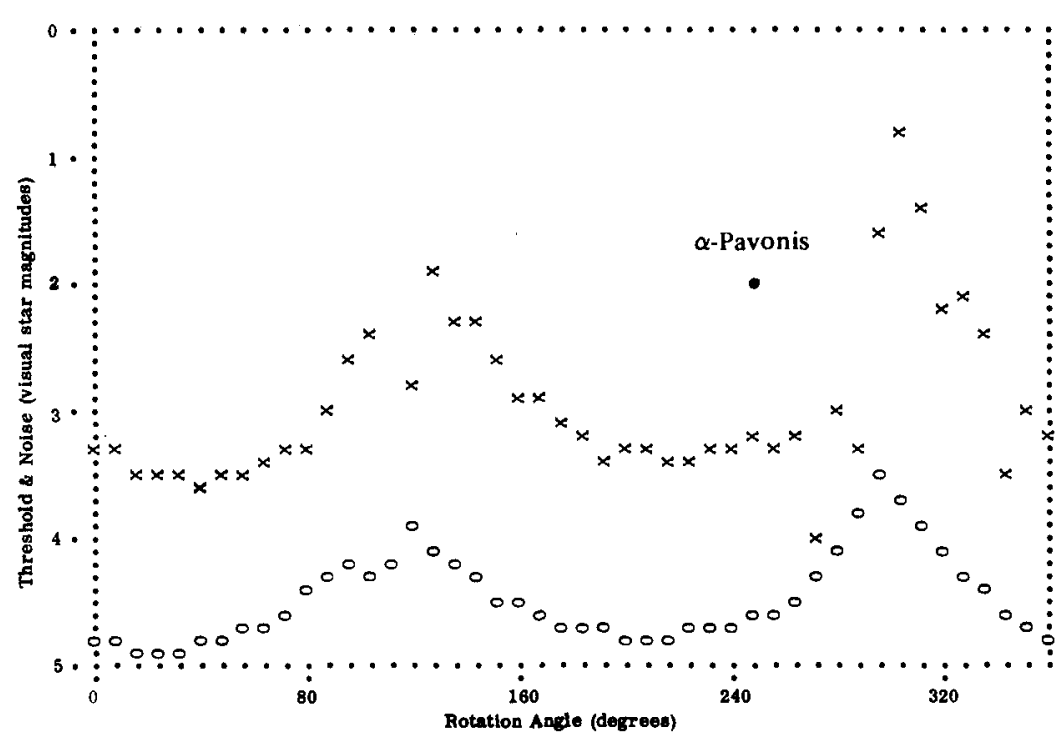

Figure 8.-Computer-generated cyclic threshold variation for Pioneer $F$ and G A/MD in the narrowband mode. Galactic longitude $=333^{\circ}$, galactic latitude $=0^{\circ}$, bandwidth $=13 \mathrm{kHz} . x$ indicates threshold, $O$ indicates noise.

TABLE V.--Probability of Detecting a Large Asteroid (a $>10$ m) During a Mission, Narrowband Mode

\begin{tabular}{r|r|r|r|r}
\hline \multirow{2}{*}{$r$} & \multicolumn{4}{|c}{$\alpha$} \\
\cline { 2 - 5 } & \multicolumn{1}{|c|}{1.75} & \multicolumn{1}{|c|}{2.0} & \multicolumn{1}{c}{2.5} & \multicolumn{1}{c}{3.0} \\
\hline $0.07 \ldots$ & 0.09 & 1.7 & 24 & $\sim 100$ \\
$0.2 \ldots$ & .24 & 4.9 & 53 & $\sim 100$ \\
$0.3 \ldots$ & .37 & 6.9 & 67 & $\sim 100$ \\
\hline
\end{tabular}

Values are in percent.

narrowband mode in terms of the probability of seeing a single large asteroid is shown in table $\mathrm{V}$.

\section{ACKNOWLEDGMENTS}

This effort was partially supported by NASA under contract NAS2-5609. The authors are indebted to Marie Wise for her assistance, patience, and forbearance.

\section{REFERENCES}

Anders, E. 1965, Fragmentation History of Asteroids. Icarus 4, 399.

Dohnanyi, J. S. 1969, Collisional Model of Asteroids and Their Debris. J. Geophys. Res.

74(10), 2531. 
Gehrels, T. 1970, Photometry of Asteroids. Surfaces and Interiors of Planets and Satellites (ed., A. Dollfus), p. 317. Academic Press, Inc. New York.

Houten, C. J. van, Houten-Groeneveld, 1. van, Herget, P., and Gehrels, T. 1970, Palomar-Leiden Survey of Faint Minor Planets. Astron. Astrophys. Suppl. Ser. 2, 339. Kessler, D. J. 1968, Upper Limit on the Spatial Density of Asteroidal Debris. AIAA J. 6(12), 2450.

Kuiper, G. P., Fujita, Y., Gehrels, T., Groeneveld, I., Kent, J., Van Biesbroeck, G., and Houten, C. J. van. 1958, Survey of Asteroids. Astrophys. J. Suppl. Ser. 3, 289.

NASA PT-204. 1970, Pioneer F Asteroid Analysis. NASA Ames Research Center.

NASA SP-8013. 1969, Meteoroid Environment Model-1969 (Near Earth to Lunar Surface).

Piotrowski, S. 1953, The Collisions of Asteroids. Acta Astron. 5, 135.

Roach, F. E., and Megill, L. R. 1961, Integrated Starlight Over the Sky. Astrophys. J. 133, 228.

Soberman, R. K., and Neste, S. L. 1971, Sisyphus Threshold Program (THRESH1). General Electric PIR 2R90-34.

\section{DISCUSSION}

GREYBER: It seems to me that a telescope using a computer-controlled imaging system, such as an image-orthicon tube, would be preferable for optical detection of asteroids from a spacecraft traversing the asteroid belt to the method chosen by Soberman, especially on a stabilized, nonspinning spacecraft. Stirling Colgate has developed such technology for the rapid detection of supernovas in external galaxies. In principle for asteroid detection one would write a computer program that would "ignore" the background of stars and search for objects moving rapidly across the field of view; i.e., planets, comets, and asteroids.

[Editorial note: The Pioneer Mission to Jupiter is described in NASA SP.268.] 